\title{
A Compound Class of Geometric and Lifetimes Distributions
}

\author{
Said Hofan Alkarni* \\ Department of Quantitative Analysis, King Saud University, Riyadh, Saudi Arabia
}

\begin{abstract}
A new lifetime class with decreasing failure rateis introduced by compounding truncated Geometric distribution and any proper continuous lifetime distribution. The properties of the proposed class are discussed, including a formal proof of its probability density function, distribution function and explicit algebraic formulae for its reliability and failure rate functions. A simple EM-type algorithm for iteratively computing maximum likelihood estimates is presented. A formal equation for Fisher information matrix is derived in order to obtaining the asymptotic covariance matrix. This new class of distributions generalizes several distributions which have been introduced and studied in the literature.
\end{abstract}

Keywords: Lifetime distributions, decreasing failure rate, Geometric distribution.

\section{INTRODUCTION}

The study of life time organisms, devices, structures, materials, etc., is of major importance in the biological and engineering sciences. A substantial part of such study is devoted to the mathematical description of the length of life by a failure distribution. Sometimes physical considerations of the failure mechanism may lead to a specific distribution but more often, the choice is made on the basis of how well the actual observations of times to failure appear to be fitted by the distribution. In reliability studies a useful tool for reducing the range of possible candidates is provided by the shape and monotonicity of the failure rate function since it reflects some of the characteristics of the mechanism leading to life conclusion.

Situations where the failure rate function decreases with time have been reported by several authors. Indicative examples are business mortality [1], failure in the air-conditioning equipment of a fleet of Boeing 720 aircrafts or in semiconductors from various lots combined [2] and the life of integrated circuit modules [3]. In general, a population is expected to exhibit a decreasing failure rate (DFR) when its behavior over time is characterized by 'work hardening' (in engineering terms) or 'immunity' (in biological terms); sometimes the broader term 'infant mortality' is used to denote the DFR phenomenon. The resulting improvement of reliability with time might have occurred by means of actual physical changes that caused self-improvement or simply it might have been due to population heterogeneity. Indeed, [2] provided that the DFR property is inherent to mixtures of distributions with constant failure rate [4] for other properties of exponential mixtures [5], demonstrated the converse for any gamma distribution with shape parameter less than one. In addition, [6] give examples illustrating that such results may hold for mixtures of distributions with rapidly increasing failure rate. A mixture of truncated geometric distribution

*Address correspondence to this author at the Department of Quantitative Analysis, King Saud University, Riyadh, Saudi Arabia; Tel: ????????????; Fax: ???????????; E-mail: salkarni@ksu.edu.sa and exponential with DFR was introduced by [7]. The exponential-Poisson (EP) distribution was proposed by [8] and generalized [9] using Wiebull distribution and the exponentiallogarithmic distribution discussed by [10]. A two-parameter distribution family with decreasing failure rate arising by mixing power-series distribution has been introduced [11]. A Weibull power series class of distributions with Poisson presented $[12,13]$ in a master degree thesis presented a class of generalized Beta distributions, Pareto power series and Weibull power series. Lately [14], obtained a class of truncated Poisson with any continuous lifetime distribution.

A further exponentiated type distribution has been introduced and studied in the literature. The exponential Weibull (EW) distribution was proposed [15] to extend the geometric exponential (GE) distribution. This distribution was also studied [16-19] introduced four more exponentiated type distributions: the exponentiated gamma, exponentiated Weibull, exponentiated Gumbel and exponentiated Fréchet distributions by generalizing the gamma, Weibull, Gumbel and Fréchet distributions in the same way that the GE distribution extends the exponential distribution. In a recent paper, [20] introduced the generalized exponential-Poisson distribution which extends the exponential-Poisson distribution in the same way that the GE distribution extends the exponential distribution.

In this paper we generalize the work of [7] to a class of several lifetime continuous distributions. This paper is organized as follow. In Section 2, the new class of geometric lifetime distributions with its probability and distribution functions are introduced. In Section 3, the corresponding survival and hazard rate functions with some of their properties are derived. In Section 4,maximum likelihood estimate of the unknown parameters are obtained based on a random sample via EM algorithm. In Section 5, the entropy for the geometric lifetime distributions class is discussed.

\section{THE CLASS}

Given $Z$, let $T_{1}, \ldots, T_{Z}$ be independent and identically distributed (iid) random variables with probability density function (pdf) given by 


$$
f_{T_{i}}(x ; \underline{\theta})=f_{T}(x ; \underline{\theta}) ; \underline{\theta}=\left(\theta_{1}, \ldots, \theta_{\mathrm{k}}\right), k \geq 1, x, \underline{\theta} \in \mathbb{R}^{+}
$$

Here, $Z$ is a zero truncated Geometric random variable with probability mass function given by

$$
f_{Z}(z ; p)=(1-p) p^{z-1}, z \in, 0<p<1,
$$

where

$Z$ and $T_{i}, i=1, \ldots, Z$ are independent random variables. Let $\mathrm{X}=\min \left(T_{1}, \ldots, T_{z}\right)$ then, the pdf of the random variable $X$ is obtained as

$$
\mathrm{f}_{\mathrm{X}}(x ; \mathrm{p}, \underline{\theta})=\frac{(1-\mathrm{p}) f_{T}(x ; \underline{\theta})}{\left[1-\mathrm{p}\left(\left(1-F_{T}(x ; \underline{\theta})\right)\right]^{2}\right.}
$$
$X$ is

and hence the cumulative distribution function (cdf) of

$\mathrm{F}_{\mathrm{X}}(x ; \mathrm{p}, \underline{\theta})=\frac{F_{T}(x ; \underline{\theta})}{1-\mathrm{p}\left(\left(1-F_{T}(x ; \underline{\theta})\right)\right.}$

The proof of the results in (1) and (2) is presented in the following theorem.

Theorem 2.1. Suppose that $\mathrm{T}_{1}, \ldots, \mathrm{T}_{Z}$ with $f_{T_{i}}(x, \underline{\theta})=f_{T}(x, \underline{\theta}), \underline{\theta}=\left(\theta_{1}, \ldots, \theta_{\mathrm{k}}\right)$ for $, k \geq 1, x, \underline{\theta} \in \mathbb{R}^{+}$ and $\mathrm{Z}$ is a zero truncated Geometric variable with probability mass function $f_{Z}(z ; p)=(1-\mathrm{p}) p^{z-1}, z \in \mathbb{N}, 0<p<1$ where $Z$ and $T_{i}, i=1, \ldots, Z$ are independent random variables. If $\mathrm{X}$ $=\min \left(\mathrm{T}_{1}, \ldots, \mathrm{T}_{z}\right)$ then the pdf and cdf of $X$ are

$\mathrm{f}_{\mathrm{X}}(x ; \mathrm{p}, \underline{\theta})=\frac{(1-\mathrm{p}) f_{T}(x ; \underline{\theta})}{\left[1-\mathrm{p}\left(\left(1-F_{T}(x ; \underline{\theta})\right)\right]^{2}\right.}$

and

$\mathrm{F}_{\mathrm{X}}(x ; \mathrm{p}, \underline{\theta})=\frac{F_{T}(x ; \underline{\theta})}{1-\mathrm{p}\left(\left(1-F_{T}(x ; \underline{\theta})\right)\right.}$

respectively.

Proof: By definition, the pdf of $X$ given $Z=z$ is

$f_{X \mid Z}(\mathrm{x} ; \underline{\theta})=\frac{\mathrm{f}_{\mathrm{X}, \mathrm{Z}}(\mathrm{x}, \mathrm{z} ; \mathrm{p}, \underline{\theta})}{\mathrm{f}_{\mathrm{Z}}(z)}=\mathrm{z} f_{T}(\mathrm{x} ; \underline{\theta})\left[1-F_{T}(\mathrm{x} ; \underline{\theta})\right]^{\mathrm{z}-1}$,

and hence the joint pdf of $X$ and $Z$ is obtained as

$\mathrm{f}_{\mathrm{X}, \mathrm{Z}}(\mathrm{x}, \mathrm{z} ; \mathrm{p}, \underline{\theta})=\mathrm{f}_{\mathrm{Z}}(z) f_{X \mid Z}(\mathrm{x} ; \underline{\theta})$

$=(1-\mathrm{p}) p^{z-1} z f_{T}(\mathbf{x} ; \underline{\theta})\left[1-F_{T}(\mathbf{x} ; \underline{\theta})\right]^{\mathrm{z}-1}$.

The marginal pdf and cdf of $\mathrm{X}$ are given by

$$
\mathrm{f}_{\mathrm{X}}(\mathrm{x} ; p, \underline{\theta})=\sum_{\mathrm{z}=1}^{\infty} \mathrm{f}_{\mathrm{X}, \mathrm{Z}}(\mathrm{x}, \mathrm{z} ; \mathrm{p}, \underline{\theta})
$$

$$
\begin{aligned}
& =(1-\mathrm{p}) f_{T}(\mathrm{x} ; \underline{\theta}) \sum_{\mathrm{z}=1}^{\infty} \mathrm{z}\left[p\left(1-F_{T}(\mathrm{x} ; \underline{\theta})\right)\right]^{z-1} \\
& =\frac{(1-\mathrm{p}) f_{T}(x ; \underline{\theta})}{\left[1-\mathrm{p}\left(\left(1-F_{T}(x ; \underline{\theta})\right)\right]^{2}\right.}
\end{aligned}
$$

and

$$
\begin{aligned}
& \mathrm{F}_{\mathrm{X}}(\mathrm{x} ; p, \underline{\theta})=\int_{0}^{x} \mathrm{f}_{\mathrm{X}}(\mathrm{x} ; p, \underline{\theta}) d x=\int_{0}^{x} \frac{(1-\mathrm{p}) f_{T}(x ; \underline{\theta})}{\left[1-\mathrm{p}((1-T(x ; \underline{\theta}))]^{2}\right.} d x \\
& =\frac{F_{T}(x ; \underline{\theta})}{1-\mathrm{p}\left(\left(1-F_{T}(x ; \underline{\theta})\right)\right.}
\end{aligned}
$$

respectively.

We denote a random variable $X$ with pdf and cdf (1) and (2) by $X \sim G L(p, \underline{\theta})$. This new class of distributions generalizes several distributions which have been introduced and studied in the literature. For instance using the probability density and its distribution function of exponential distribution in (1), we obtain the exponential geometric distribution [7] and using Wiebull probability density and its distribution function gives Wiebull geometric distribution [21]. The model is obtained under the concept of population heterogeneity (through the process of compounding). An interpretation of the proposed model is as follows: a situation where failure (of a device for example) occurs due to the presence of an unknown number, $\mathrm{Z}$, of initial defects of same kind (a number of semiconductors from a defective lot, for example). According to [7], the Ts represent their lifetimes and each defect can be detected only after causing failure, in which case it is repaired perfectly. Then the distributional assumptions given earlier lead to any of the GL distributions for modeling the time to the first failure $X$.

Table 1 shows the probability function and the distribution function for some lifetime distributions.

Some of the other lifetime distributions are excluded from this table since they do not have nice forms such as Gamma and lognormal distributions although they still can be applied in this class numerically.

The qth quantile $x_{q}$ of the GL distribution, the inverse of the distribution function $\mathrm{F}_{\mathrm{X}}\left(x_{q}\right)=\mathrm{q}$ is the same as the inverse of the distribution function $\mathrm{F}_{\mathrm{T}}\left(x_{q}\right)=\frac{q(1-p)}{1-p q}$ for any continuous lifetime with distribution function $\mathrm{F}_{\mathrm{T}}($.).

\section{SURVIVAL AND HAZARD FUNCTIONS}

Since the GL is not a part of the exponential family, there are no simple form for moments see for instant (Kus[8]) for the exponential case. Survival function (also known reliability function) ( $\mathrm{sf}$ ) and hazard function (known as failure rate function) (hf) for the GL class are given in the following theorem. 
Table 1. Probability and Distribution Functions

\begin{tabular}{|c|c|}
\hline $\mathrm{f}_{\mathrm{X}}(x ; \mathrm{p}, \underline{\theta})$ & $\mathrm{F}_{\mathrm{X}}(x ; \mathrm{p}, \underline{\theta})$ \\
\hline \hline Exponential $\frac{(1-p) \lambda e^{-\lambda x}}{\left[1-p e^{-\lambda x}\right]^{2}}$ & $\frac{1-e^{-\lambda x}}{1-p e^{-\lambda x}}$ \\
\hline Weibull $\frac{(1-p) \alpha \beta(\beta x)^{\alpha-1} e^{-(\beta x)^{\alpha}}}{\left[1-p e^{-(\beta x)^{\alpha}}\right]^{2}}$ & $\frac{1-e^{-(\beta x)^{\alpha}}}{1-p e^{-(\beta x)^{\alpha}}}$ \\
\hline Rayleigh $\frac{(1-p) \theta^{2} x e^{-\frac{\theta^{2}}{2} x^{2}}}{-\theta^{2}} x^{2}$ & $\frac{1-e^{-\frac{\theta^{2}}{2} x^{2}}}{11-p e^{2}}$ \\
\hline Pareto $\frac{(1-p) \gamma(1+x)^{-\frac{\theta^{2}}{2} x^{2}}}{\left[(1+x)^{\gamma}-p\right]^{2}}$ & $\frac{(1+x)^{\gamma}-1}{(1+x)^{\gamma}-p}$ \\
\hline
\end{tabular}

Theorem 3.1 Suppose that $T_{1}, \ldots, T_{Z}$ are independent random variables with

$f_{T_{i}}(x, \underline{\theta})=f_{T}(x, \underline{\theta}), \underline{\theta}=\left(\theta_{1}, \ldots, \theta_{\mathrm{k}}\right)$

for , $k \geq 1, x, \underline{\theta} \in \mathbb{R}^{+}$and $\mathrm{Z}$ is a zero truncated Geometricrandom variable with probability mass function

$f_{Z}(z ; p)=(1-\mathrm{p}) p^{z-1}, z \in \mathbb{N}, 0<p<1$

where $Z$ and $T_{i}, i=1, \ldots, Z$ are independent random variables. If $X=\min \left(\mathrm{T}_{1}, \ldots, \mathrm{T}_{\mathrm{Z}}\right)$, then the sf and hf of $X$ are

$s_{X}(x ; p, \underline{\theta})=\frac{(1-p) s_{T}(x ; \underline{\theta})}{1-p s_{T}(x ; \underline{\theta})}$

and

$h_{X}(x ; p, \underline{\theta})=\frac{f_{T}(x ; \underline{\theta})}{s_{T}(x ; \underline{\theta})\left(1-\mathrm{p} s_{T}(x ; \underline{\theta})\right)}$

respectively, where $S_{T}(x ; \underline{\theta})$ is the survival function of any continuous lifetime distribution.

Proof: Using (1) and (2), survival function (also known reliability function) and hazard function (known as failure rate function) for the GL class are given respectively by

$$
\begin{aligned}
& s_{X}(x ; p, \underline{\theta})=1-\mathrm{F}_{X}(x ; \mathrm{p}, \underline{\theta})=1-\frac{F_{T}(x ; \underline{\theta})}{1-\mathrm{p}\left(\left(1-F_{T}(x ; \underline{\theta})\right)\right.} \\
& =\frac{s_{T}(x ; \underline{\theta})-p s_{T}(x ; \underline{\theta})}{1-p s_{T}(x ; \underline{\theta})}=\frac{(1-p) s_{T}(x ; \underline{\theta})}{1-p s_{T}(x ; \underline{\theta})}
\end{aligned}
$$

and

\begin{tabular}{|c|c|}
\hline$s_{X}(x ; \mathrm{p}, \underline{\theta})$ & $h_{X}(x ; \mathrm{p}, \underline{\theta})$ \\
\hline Exponential $\frac{(1-p) e^{-\lambda x}}{1-p e^{-\lambda x}}$ & $\frac{\lambda}{1-\mathrm{p} e^{-\lambda x}}$ \\
\hline Weibull $\frac{(1-p) e^{-(\beta x)^{\alpha}}}{1-\mathrm{p} e^{-(\beta x)^{\alpha}}}$ & $\frac{\alpha \beta(\beta x)^{\alpha-1}}{1-\mathrm{p} e^{-(\beta x)^{\alpha}}}$ \\
\hline Rayleigh $\frac{(1-p) e^{-\frac{\theta^{2}}{2} x^{2}}}{1-p e^{-\frac{\theta^{2}}{2} x^{2}}}$ & $\frac{\theta^{2} x}{1-\mathrm{p} e^{-\frac{\theta^{2}}{2} x^{2}}}$ \\
\hline Pareto $\frac{1-p}{(1+x)^{\gamma}-p}$ & $\frac{\gamma}{(1+\boldsymbol{x})^{\gamma}-\boldsymbol{p}(1+\boldsymbol{x})^{1-\gamma}}$ \\
\hline $\begin{array}{l}h_{X}(x ; p, \underline{\theta}) \\
=\frac{\mathrm{f}_{\mathrm{X}}(x ; \mathrm{p}, \underline{\theta})}{s_{X}(x ; p, \underline{\theta})}=\frac{(1-\mathrm{p}) f_{T}(x ; \underline{\theta})}{\left[1-p s_{T}(x ; \underline{\theta})\right]^{2}}\end{array}$ & $\frac{1-p s_{T}(x ; \underline{\theta})}{(1-p) s_{T}(x ; \underline{\theta})}$ \\
\hline$=\frac{f_{T}(x ; \underline{\theta})}{s_{T}(x ; \underline{\theta})\left(1-p s_{T}(x ; \underline{\theta})\right)}$ & \\
\hline
\end{tabular}

Table 2. Survival and Hazard Functions

Table 2 summarizes the survival functions and hazard rate functions for some distributions of the class.

The hazard function for GL class is decreasing because the DFR property follows from the result of Barlow et al. (1963) [22] on mixture.

\section{ESTIMATION}

In what follows, we discuss the estimation of the GL class parameters. Let $x_{1}, \ldots, x_{\mathrm{n}}$ be a random sample with observed values $x_{1}, \ldots, x_{\mathrm{n}}$ from a GL distribution with parameters $p$ and $\underline{\theta}$. Let $\mathrm{E}=(p, \underline{\theta})$ be the parameter vector. The $\log \log$-liketihood function based on the observed random sample size of $n, y_{\mathrm{obs}}=\left(x_{1}, \ldots, x_{\mathrm{n}}\right)$ is obtained by

$$
\begin{aligned}
& \ell\left(p, \underline{\theta} ; y_{\text {obs }}\right)= \\
& n \log (1-\mathrm{p})+\sum_{i=1}^{n} \log f_{T}\left(\mathrm{x}_{i} ; \underline{\theta}\right)-2 \sum_{i=1}^{n} \log \left(1-p\left(1-F_{T}\left(\mathrm{x}_{i} ; \underline{\theta}\right)\right)\right)
\end{aligned}
$$

and the associated score function is given by $U_{n}(\underline{\mathrm{E}})=\left(\frac{\partial \ell}{\partial p}, \frac{\partial \ell}{\partial \theta_{1}}, \ldots, \frac{\partial \ell}{\partial \theta_{k}}\right)$, where

$\frac{\partial \ell\left(p, \underline{\theta} ; y_{o b s}\right)}{\partial p}=-\frac{n}{1-p}+2 \sum_{i=1}^{n} \frac{1-F_{T}\left(\mathrm{x}_{i} ; \underline{\theta}\right)}{1-p\left(1-F_{T}\left(\mathrm{x}_{i} ; \underline{\theta}\right)\right)}$

and

$\frac{\partial \ell\left(\underline{\grave{e}} ; y_{o b s}\right)}{\partial \theta_{i}}=\sum_{i=1}^{n} \frac{1}{f_{T}\left(\mathrm{x}_{i} ; \underline{\theta}\right)} \frac{\partial f_{T}\left(\mathrm{x}_{i} ; \underline{\theta}\right)}{\partial \theta_{i}}-$ 


$$
2 p \sum_{i=1}^{n} \frac{\frac{\partial F_{T}\left(\mathrm{x}_{i} ; \underline{\theta}\right)}{\partial \theta_{i}}}{1-p\left(1-F_{T}\left(\mathrm{x}_{i} ; \underline{\theta}\right)\right)}, i=1, \ldots, k
$$

The maximum likelihood estimates (MLE) of E, say E, is obtained by solving the nonlinear system $U_{n}(\Theta)=0$. The solution of this nonlinear system of equations has not a closed form, but can be found numerically by using software such as MATHEMATICA, MAPLE, Ox and R.

For interval estimation and hypothesis tests on model parameters, we require the information matrix. The $(k+1) \times(k+1)$ information matrix is given by

$$
I_{n}(\Theta)=\left(\begin{array}{cc}
l_{p p}^{\prime \prime} & l_{p \theta_{i}}^{\prime \prime} \\
l_{\theta_{i} p}^{\prime \prime} & l_{\theta_{i} \theta_{i}}^{\prime \prime}
\end{array}\right)
$$

where the elements of $I_{n}(\Theta)$ are the second partial derivatives of (5) and (6). Under the regular conditions stated in (Cox and Hinkley [23]), that are fulfilled for our model whenever the parameters are in the interior of the parameter space, we have that the asymptotic distribution of $\sqrt{n}(\hat{\Theta}-\Theta)$ is multivariate normal $N_{k+1}\left(0, k(\Theta)^{-1}\right)$, where $k(\Theta)=\lim _{n \rightarrow \infty} n^{-1} I_{n}(\Theta)$ is the unit information matrix.

\subsection{EM Algorithm}

Based on the underlying distribution, the maximum likelihood estimation of the parameters can be found analytically using an EM algorithm. Newton-Raphson algorithm is one of the standard methods to determine the MLEs of the parameters. To employ the algorithm, second derivatives of the log-likelihood are required for all iteration. EM algorithm is a very powerful tool in handling the incomplete data problem (Dempster, Laird and Rubin [24, 25]. It is an iterative method by repeatedly replacing the missing data with estimated values and updating the parameter estimates. It is especially useful if the complete data set is easy to analyze. As pointed out by [26], the EM algorithm will converge reliably but rather slowly (as compared to the Newton-Raphson method) when the amount of information in the missing data is relatively large. Recently, EM algorithm has been used by several authors such as [7, 27-30].

To estimate $\Theta$, EM algorithm is a recurrent method such that each step consists of an estimate of the expected value of a hypothetical random variable and later maximizes the log-likelihood of the complete data. Let the complete data be $X_{1}, \ldots, X_{n}$ with observed values $x_{1}, \ldots, x_{n}$ and the hypothetical random variable $Z_{1}, \ldots, Z_{n}$. The joint probability function is such that the marginal density of $X_{1}, \ldots, X_{n}$ is the likelihood of interest. Then, we define a hypothetical complete-data distribution for each $\left(X_{i}, Z_{i}\right)^{T}, i=1, \ldots, n$,

With a joint probability function in the form
$\mathrm{f}_{\mathrm{X}, \mathrm{Z}}(\mathrm{x}, \mathrm{z} ; \Theta)=(1-\mathrm{p}) p^{z-1} z f_{T}(\mathrm{x} ; \underline{\theta})\left[1-F_{T}(\mathrm{x} ; \underline{\theta})\right]^{\mathrm{z}-1}$

with $x, \underline{\theta} \in \mathbb{R}^{+}, 0<p<1$ and $z \in \mathbb{N}$. Thus, it is straightforward to verify that the Estep of an EM cycle requires the computation of the conditional expectation of $\left(Z \mid X ; \Theta^{(r)}\right)$, where $\Theta^{(r)}=\left(\mathrm{p}^{(r)}, \underline{\theta}^{(r)}\right)$ isthe current estimate (in the rth iteration) of $\dot{\mathrm{E}}$. The EM cycle is completed with M-step, which is complete data maximum likelihood over $(\Theta)$, with the missing Z's replaced by their conditional expectations $E(\mathrm{Z} \mid \mathrm{X} ; \grave{\mathrm{E}})$ (Adamidis and Loukas [7]), where

$$
f_{Z \mid X}(z)=z\left[p\left(1-F_{T}(x ; \underline{\theta})\right)\right]^{z-1}\left[\left(1-\mathrm{p}\left(1-F_{T}(x ; \underline{\theta})\right)\right]^{2}\right.
$$

and its expected value is

$$
E(Z \mid X)=\frac{1-\mathrm{p}\left(1-F_{T}(x ; \underline{\theta})\right)}{p^{2}\left(1-F_{T}(x ; \underline{\theta})\right)^{2}} .
$$

\section{ENTROPY FOR THE CLASS}

If $X$ is a random variable having an absolutely continuous cumulative distribution function $F x(x)$ and probability distribution function $f_{x}(x)$ then the basic uncertainty measure for distribution $F$ (called the entropy of $F$ ) is defined as $H_{X}(X)=-E(\log f(x))$, hence the general entropy form for the GL class is given

$$
\begin{aligned}
& H_{X}(X)=-E(\log f(X))=H_{T}(X)-\log (1-p)+ \\
& 2 E\left[\log \left(1-\mathrm{p}\left(1-F_{T}(x ; \underline{\theta})\right)\right)\right] \triangleleft
\end{aligned}
$$

where $H_{T}(X)$ is the entropy of any lifetime distribution in the class. Note that as $p$ increases the $H_{X}(X)$ increases too which is very logical since the increase of probability of accidents increases the entropy.

Statistical entropy is a probabilistic measure of uncertainty or ignorance about the outcome of a random experiment, and is a measure of a reduction in that uncertainty. Since [31] pioneering work on the mathematical theory of communication, entropy has been used as a major tool in information theory and in almost every branch of science and engineering. Numerous entropy and information indices, among them the Renyi entropy, have been developed andused in various disciplines and contexts. Information theoretic principles and methods have become integral parts of probability and statistics and have been applied in various branches of statistics and related fields.

\section{CONFLICT OF INTEREST}

The authors confirm that this article content has no conflicts of interest.

\section{ACKNOWLEDGEMENT}

The author is highly grateful to the deanship of scientific research at King Saud University represented by the research 
center at college of business administration for supporting this research financially.

\section{REFERENCES}

[1] K. S. Lomax, "Business failures: another example of the analysis of failure data", J. Am. Stat. Assoc. vol. 49, pp. 847-852, 1954.

[2] F.Proschan,"Theoretical explanation of observed decreasing failure rate", Technometrics, vol. 5, pp. 375-383, 1963.

[3] S.C.Saunders, and J. M. Myhre,"Maximum likelihood estimation for two-parameter decreasing hazard rate distributions using censored data", J. Am. Stat. Assoc., vol. 78, pp. 664-673, 1983.

[4] F. McNolty, J. Doyle, and E.Hansen,"Properties of the mixed exponential failure process", Technometrics., vol. 22, pp. 555-565, 1980.

[5] L. J.Gleser, "The gamma distribution as a mixture of exponential distributions", J. Am. Stat., vol. 43, pp. 115-117, 1989.

[6] J. Gurland and J. Sethuraman, "Reversal of increasing failure rates when pooling failure data", Technometrics, vol. 36, pp. 416-418, 1994.

[7] K. Adamidis, and S. Loukas,"A lifetime distribution with decreasing failure rate", Stat. Probab. Lett., vol.39, pp.35-42, 1998.

[8] C. Kus, "A new lifetime distribution", Comput. Stat. Data Anal., vol. 51, pp. 4497-4509, 2007.

[9] F. Hemmati, E. Khorram, and S. Rezakhah, "A new three- parameter distribution", J. Stat. Plan. Inference, vol. 141, pp. 2255-2275, 2011.

[10] R. Tahmasbi, and S. Rezaei, "A two-parameter lifetime distribution with decreasing failure rate", Comput. Stat. Data Anal., vol. 52, pp. 3889-3901, 2008.

[11] M. Chahkandi, and M. Ganjali, "On some lifetime distributions with decreasing failure rate", Comput. Stat. Data Anal., vol. 53, pp. 4433-4440, 2009.

[12] A. Morais, and W. Barreto-Souza, "Acompound class of Weibulland power series distributions", Comput. Stat. Data Anal.,, vol. 55, pp. 1410-1425, 2011.

[13] A.L. Morais, "A class of generalized beta distributions, pareto power series and weibull power series", M.S. thesis, Universidade Federal de Pernambuco, Recife-PE, Brasil, 2009.

[14] S. Alkarni, and A. Oraby, "A compound class of Poisson and lifetime distributions", J. Stat. Appl. Probab., vol. 1, pp. 45-51, 2012.

[15] G.S. Mudholkar, and D.K.Srivastava,"ExponentiatedWeibull family for analyzing bathtub failure-rate data", IEEE. Trans. Reliab., vol. 42, pp. 299-302, 1993.
[16] G.S. Mudholkar, D.K.Srivastava, and M. Freimer,"The exponentiated Weibull family", Technometrics., vol. 37,pp. 436-445, 1995.

[17] G. S. Mudholkar, and A. D. Hutson, "The exponentiated weibull family: Some properties and a flood data application", Commun. Stat. Theory Methods, vol. 25, pp. 3059-3083, 1996.

[18] M.M.Nassar, and F. H. Eissa, "On the exponentiatedWeibull distribution", Commun. Stat. Theory Methods, vol. 32, pp. 13171336, 2003.

[19] S.Nadarajah, and S. Kotz, "The beta exponential distribution", Reliab. Eng. Syst. Saf., vol. 91, pp. 689-697, 2006.

[20] W. Barreto-Souza, and F. Cribari-Neto, "A generalization of the exponential-Poisson distribution", Stat. Probab. Lett., vol. 79, pp. 2493-2500, 2009.

[21] R.B. Silva, W. Barreto-Souza, and G.M.Cordeiro, "A new distribution with decreasing, increasing and upside-down bathtub failure rate", Comput. Stat. Data Anal., vol. 54, pp. 935-944, 2010.

[22] R. E. Barlow, A.W.Marshall, and F.Proschan, "Properties of probability distributions with monotone hazard rate", Ann. Math. Stat., vol. 34, pp. 375-389, 1963.

[23] D. R. Cox, and D.V. Hinkley, Theoretical Statistics, Chapman and Hall: London, 1974.

[24] A. P. Dempster, N. M. Laird, and D. B. Rubin, "Maximum likelihood from incomplete data via the EM algorithm ", J. Roy. Stat. Soc. Ser. B. Stat. Methdol., vol. 39, pp. 1-38, 1977.

[25] G. J. McLachlan and T. Krishnan, "The EM Algorithm and Extension", Wiley: NewYork, 1997.

[26] R. J. A. Little, and D. B. Rubin, "Incomplete data", In: Encyclopedia of Statistical Sciences, S. Kotz, and N.L. Jhonson, Eds., vol. 4, Wiley: NewYork, 1983.

[27] K. Adamidis,"An EM algorithm for estimating negative binomial parameters", Aust. N. Z Stat., vol. 41, no. 2, pp. 213-221, 1999.

[28] H.K.T. Ng, P. S. Chan, and N. Balakrishnan, "Estimation of parameters from progressively censored data using EM algorithm", Comput. Stat. Data Anal., vol. 39, pp. 371-386, 2002.

[29] D. Karlis,"An EM algorithm for multivariate Poisson distribution and related models", J. Appl. Stat., vol. 301, pp. 63-77, 2003.

[30] K. Adamidis, T. Dimitrakopoulou, and S. Loukas, "On an extension of the exponential-geometric distribution", Stat. Probab. Lett., vol.73, pp. 259-269, 2005.

[31] E.Shannon, "A mathematical theory of communication", Bell. Sys. Tech. J., vol. 27, pp. 379-432, 1948.

\begin{tabular}{lll}
\hline Received: December 12, 2012 & Revised: January 02, 2013 & Accepted: January 14, 2013
\end{tabular}

(C) Said Hofan Alkarni; Licensee Bentham Open.

This is an open access article licensed under the terms of the Creative Commons Attribution Non-Commercial License (http://creativecommons.org/licenses/by-nc/3.0/) which permits unrestricted, non-commercial use, distribution and reproduction in any medium, provided the work is properly cited. 